\title{
A MULTI-AGENT TRAFFIC SIMULATION FRAMEWORK FOR EVALUATING THE IMPACT OF TRAFFIC LIGHTS
}

\author{
Raul Cajias, Antonio Gonzalez-Pardo and David Camacho \\ Departamento de Ingeniería Informática, Escuela Politécnica Superior, Universidad Autónoma de Madrid \\ C/Francisco Tomás y Valiente 11, 28049 Madrid, Spain \\ \{raul.cajias, antonio.gonzalez, david.camacho\}@uam.es
}

\begin{abstract}
Keywords: Multi-agent simulation, Swarm computing, Traffic optimization.
Abstract: The growing of the number of vehicles cause serious strains on road infrastructures. Traffic jams inevitably occur, wasting time and money for both cities and their drivers. To mitigate this problem, traffic simulation tools based on multiagent techniques can be used to quickly prototype potentially problematic scenarios to better understand their inherent causes. This work centers around the effects of traffic light configuration on the flow of vehicles in a road network. To do so, a Multi-Agent Traffic Simulation Framework based on Particle Swarm Optimization techniques has been designed and implemented. Experimental results from this framework show an improvement in the average speed obtained by traffic controlled by adaptive over static traffic lights.
\end{abstract}

\section{INTRODUCTION}

Traffic flow models help design dynamic control like the ones just described. Flow models are typically categorized by their level of detail in three broad classes: microscopic, mesoscopic and macroscopic (Hoogendoorn, 2001). Microscopic models describe both space and time behavior of the system's entities (vehicles and drivers) as well as their interactions at a high level of detail. Macroscopic models describe traffic behavior through high-level terms like flowrate, density and velocity. Finally, mesoscopic models consider dynamical properties that are simple enough to be simulated for long time.

The work presented in this paper is an initial study on Traffic Simulation. Agents have SWARMlike characteristics in that they lack a reasoning process. The main contribution of this work is related to the implementation of a configurable, agentbased traffic simulation framework, based on the METANET model. We model traffic flow by creating of lightweight agents using a time series distribution, enabling us to treat traffic jams as a particle swarm optimization problem (Eberhart et al., 2001). We use this approach to find dynamic traffic control configurations that will yield smooth flow of traffic at any given time. Finally, the paper shows some experimental results about how the behavior of traffic lights can affect jams in the network, depending on the traffic- flow distribution of the road.

(Burmeister et al., 1997) highlights the advantages of using Multi-Agent Systems (MAS) for traffic simulations. MAS has also been used for microscopic traffic simulation where different characteristics of the population parametrized for the model. (Zhang et al., 2005) uses a MAS for simulating single-lane roads and provide agents with a decision tree that allows them to adapt their velocity and acceleration depending on environmental constraints and desired velocity. (Ehlert and Rothkrantz, 2001), defines profiles of agents and analyses the impact of of agent behavior to the system. The two profiles defined are: fast and aggressive or slow and careful. The overall theme explored in these works are how different agent behavior impact road traffic. Furthermore, the agents fall in the category of Belive-Desire-Intention (BDI), since they have a complex and variable behavior that changes based on their surroundings and goals.

Traffic network systems such as UTOPIA-SPOT, (Mauro and Taranto, 1989), and SCOOT, (Robertson and Bretherton, 1991), integrate a number of different traffic network models to provide an urban traffic management solution through the coordinated operation of traffic signals to smooth the flow of traffic and increase circulation in cities. The use of multiagent systems for the modeling of traffic has been studied among other works in (Radecky and Gajdos, 2008) and (Xiao-Fan Zhi, 2008). (M. van den 
Berg and Hellendoorn, 2003) on the other hand, uses the METANET model (Messmer and Papageorgiou, 1990), a macroscopic model for mixed urban and freeway traffic networks, that provides integrated controls for traffic flow by implementing a model predictive control.

\section{DESCRIPTION OF THE MULTI-AGENT MODEL}

In this section a description of the Multi-agent System modeled is provided. In order to understand it correctly, the different agents and the environment are described.

\subsection{The Simulation Environment}

Road networks are modeled as a weighted directed graph $N=G(V, E)$, with source and sink $\{s, t\} \in V$, and roads $e \in E$ of $N$. The capacity of an edge is a mapping $c: E \rightarrow \mathbb{R}^{+}$, denoted by $c_{u v}$. Furthermore, the flow of the network is a mapping $f: E \rightarrow \mathbb{R}^{+}$ denoted by $f_{u v}$, subject to the following constraints:

1. Capacity constraint. For all $u, v \in V$, we require $f_{u v} \leq c_{u v}$.

2. Conservation of flows. For all $u \in V-\{s, t\}$, we require $\sum_{v, u} f_{v u}=0 \forall v, u \in E$

3. Skew Symmetry. For all $u, v \in V$, we require $f_{u v}=$ $-f_{v u}$

Road capacity is a function of the road's width (number of lanes) and length. Of the two, only the number of lanes in a road follows specific rules that can serve to differentiate one road from another.

Given equal-length road segments, road capacity translates to the number of vehicles that can fit that segment, multiplied by the number of segments in a road. Thus, by toggling the number of vehicles that fit in a single section, new road types can be defined. This implementation has the added benefit that traffic accidents can easily be modeled: By decreasing the maximum number of vehicles in a single segment for a arbitrary number of steps, the impact of an accident in roads can be easily simulated.

\subsection{Vehicle and Traffic Light Agents}

The system is composed of two types of agents: vehicles and traffic lights. These agents interact with each other by keeping track of local information available to them, and acting accordingly.
Upon creation, vehicles are assign a shortest distance trajectory between two arbitrary points in the road network, which will remain unchanged throughout the life of the agent. These agents can be created following a distribution function or at a constant rate. As they travel through the road network, agents require local information to decide whether to move or stay put. This information is made available to them by consulting the status blackboard maintained by each road. As they traverse through the network, vehicles can consult the status of the traffic light and the number of cars currently residing at the immediate next road section and use this information to move forward or stop.

Traffic light agents (TLAs) can have two states: red and green. How long a red-to-green cycle lasts, as well as how much of that time is allocated for each state can be static or dynamic. The platform provides a static agent implementation out-of-the box, and a mechanism for attaching custom dynamic agents. TLAs can be located at either street endpoints or intersections.

A type of dynamic TLA developed was an agent that would measure the current state of the roads leading to it, and split the total duration of it's red-to-green cycle to favor the most impacted road. As the current implementation only supports intersections between two roads, this version of an adaptable TFA only takes care of this case. The process for this agent follows:

1. Traffic light compares the traffic flow of each road. The following equation defines the traffic flow of a road:

$$
f(\text { road })=\frac{n_{\text {Segments }} * n_{\text {VehiclesPerSegment }}}{n_{\text {VehiclesOnRoad }}}
$$

In this equation, $n_{\text {Segments }} * n_{\text {VehiclesPerSegment }}$ defines the maximum number of vehicles allowed in a road, and $n_{\text {VehiclesOnRoad }}$ is the real number of vehicles currently on the road.

2. The traffic flow of both roads are compared to know what road has more traffic.

3. Finally, the split of the traffic light is adjusted in the way that the road with more traffic takes advantage. The new split is the percentage of traffic flow that travels through the road with more traffic. The equation used is the following:

$$
\text { NewSplit }=\frac{\max \left(f\left(\operatorname{road}_{1}\right)\right)}{\sum_{i=1}^{2} f\left(\operatorname{road}_{i}\right)}
$$

Where $\max \left(f\left(\operatorname{road}_{1}\right)\right)$ represents the traffic flow of the road with more traffic, and $\sum_{i=1}^{2} f\left(\right.$ road $\left._{i}\right)$ is the sum of the traffic flow of both roads. 


\section{DESIGNING A SIMULATION}

Simulations are defined using an XML simulation description file. This file is used to describe all aspects of the simulation, from the road network, types of roads, the trajectories vehicles should take, to vehicle distribution and location and type of traffic lights to be used.

Roads are defined as a series of points in a cartesian plane, an orientation and one of two directions \{one-way, two-way\}. Trajectories can be set to be either street endpoints or intersections. In each, the percentage of traffic to start from and finish in start and finish road nodes is specified. This allows the freedom to play with different traffic flow configurations. Street-light agents can be positioned in street endpoints or at intersections, and the duration of a whole red-to-green state cycle can be set as well.

Once the simulation is completed, an output file is created for each trajectory vehicles took to reach their desired destinations. These files are also in XML format, allowing a wide range of statistics to be extracted from each experiment.

\section{EXPERIMENTAL RESULTS}

The simulation framework was used to observe the impact of both static and dynamic TLAs on flow. To do so, we measure the average speed attained by vehicles traveling down a given trajectory, and compare like trajectories with different configuration of each TLA.

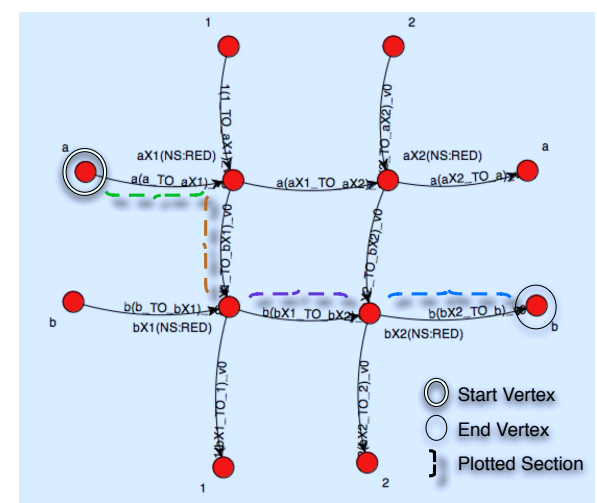

Figure 1: A description of the road network used in this experiment batch.

In these experiments (Figure 1), two separate simulation batteries where defined: one set for static and one for adaptive lights. Three different situations were used to simulate the road had a low traffic flow

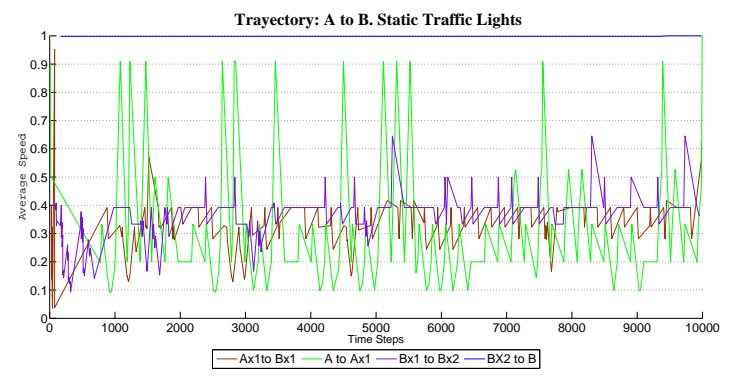

Figure 2: A plot of the average speeds attained by vehicles traversing four roads in the trajectory $\left\{a_{\text {start }} \rightarrow b_{\text {finish }}\right\}$.

(only $10 \%$ of the traffic was traveling through the road), the road had a normal flow with $50 \%$ of the traffic, and finally, the road was congested (with $90 \%$ of the traffic). Figures 2 and 3 results of using an adaptive versus a static traffic light to regulate the traffic of roads in a single trajectory with $90 \%$ of the traffic.

Figure 2 shows the average speeds of the different roads for trajectory $\left\{a_{\text {start }} \rightarrow b_{\text {finish }}\right\}$. For vehicles traversing the network of traffic lights, periodic spikes and valleys begin to populate each road's average velocity plot. Traffic lights act as a flow filter, smoothing out traffic velocity in the system at each intersection, until traffic finally exits the network at maximum speed. Towards the end of the simulation, periodic patterns in velocity suggest that the system reaches some equilibrium, a result not at all surprising given the passive nature of the interactions in the system.

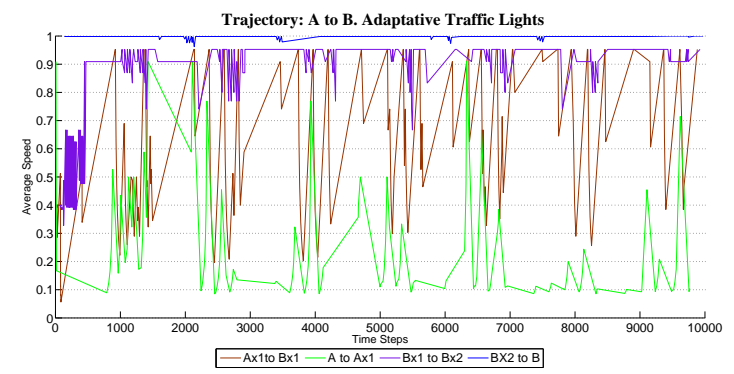

Figure 3: A plot of the average speeds attained by vehicles traversing four roads in the trajectory $\left\{a_{\text {start }} \rightarrow b_{\text {finish }}\right\}$.

Introducing adaptive traffic lights into the system yields a more interesting average graph (Figure 3 ). Periodicity is still a defining characteristic of the plot, but more interesting patterns are observed. While quite simple in nature, the attempt by early traffic lights to maximize vehicle flow, causes a ripple effect to later traffic lights that result in chaotic patterns in the average road velocity through time. In general, the average road velocity experiences an improvement over the static traffic light experiment. 
These results coupled with the quick simulation turnaround time yielded by this framework, suggest that there is ample room for exploring the impact of more complex traffic light agents, and that such territory can be covered with the help of this tool.

\section{CONCLUSIONS AND FUTURE WORK}

We propose a MAS simulation framework for urban traffic simulation, using a swarm agent model. Simulation designers are free to configure road networks of arbitrary complexity, by customizing road width, geometry and intersection with other roads, as well as define source and sink locations for vehicles.

We have tested the simulation using static and dynamic traffic light agents, in order to observe the impact to network flow. Static TLAs cycle through their states at a constant rate, while the dynamic TLA implemented attempts to optimize the average speed of the vehicles in the network by favoring roads with higher traffic flow. The average vehicle speed was higher for networks with the dynamic TLAs, which suggest that such agents may be key elements in a wider flow optimization strategy, under more demanding traffic scenarios.

Further work is planned to test the performance of these two traffic light agents in network graphs, orders of magnitude larger that the ones used in these experiments, and the implementation of a time series vehicle generation function that better mimics real traffic flow scenarios. We are also interested in allowing cars to adapt their trajectory to optimize their movement through the network, based on local information available to them. Finally there is strong motivation to use automatic discovery methods such as genetic algorithms, to find combinations of different types of traffic light agents in a network graph, that could reach sub-optimal network flows.

\section{ACKNOWLEDGEMENTS}

This work has been supported by the Spanish Ministry of Science and Innovation. Grant TIN201019872.

\section{REFERENCES}

Burmeister, B., Haddadi, A., and Matylis, G. (1997). Application of multi-agent systems in traffic and trans- portation. IEE Proceedings - Software, 144(1):51 60.

Eberhart, R. C., Shi, Y., and Kennedy, J. (2001). Swarm Intelligence (The Morgan Kaufmann Series in Artificial Intelligence). Morgan Kaufmann.

Ehlert, P. A. and Rothkrantz, L. J. (2001). Microscopic traffic simulation with reactive driving agents. In IEEE Intelligent Transportation Systems Conference Proceedings, pages $861-866$.

Hoogendoorn, S. P. (2001). State-of-the-art of vehicular traffic flow modelling. 215(4):283 - 303 .

M. van den Berg, A. Hegyi, B. D. S. and Hellendoorn, J. (2003). A macroscopic traffic flow model for integrated control of freeway and urban traffic networks. In Proceedings of the 42nd IEEE Conference on Decision and Control, pages 2774-2779, Maui, Hawaii.

Mauro, V. and Taranto, C. D. (1989). Utopia. In Proceedings of the 6th IFAC/IFIP/IFORS Symposium on Control, Computers, Comunication on Transportation, pages 245-252, Paris, France.

Messmer, A. and Papageorgiou, M. (1990). Metanet: A macroscopic simulation program for motorway networks. In Traffic Engineering and Control, volume 31, pages 466-470.

Radecky, M. and Gajdos, P. (2008). Intelligent agents for traffic simulation. In SpringSim '08: Proceedings of the 2008 Spring simulation multiconference, pages 109,115, San Diego, CA, USA. Society for Computer Simulation International.

Robertson, D. and Bretherton, R. (1991). Optimization networks of traffic signals in real time-the scoot method. 40(1):11-15.

Xiao-Fan Zhi, Zhi-Cheng, L. S.-Y. Z. Y.-p. Z. (2008). An intersection-centric priority algorithm for traffic simulation based on multi-agents. volume 1, pages 189194. IEEE Computer Society.

Zhang, F., Li, J., and Zhao, Q. (2005). Single-lane traffic simulation with multi-agent system. In IEEE Conference on Intelligent Transportation Systems, pages $1183-1187$. 